\title{
Marc AUGÉ, Pour une anthropologie de la mobilité.
}

Paris, Payot \& Rivages, coll. Manuels Payot, 2009, 91 p.

\section{Pierre Morelli}

\section{OpenEdition}

\section{Journals}

Édition électronique

URL : http://journals.openedition.org/questionsdecommunication/256

DOI : 10.4000/questionsdecommunication.256

ISSN : 2259-8901

\section{Éditeur}

Presses universitaires de Lorraine

\section{Édition imprimée}

Date de publication : 30 juin 2010

ISBN : 978-2-8143-0024-8

ISSN : 1633-5961

\section{Référence électronique}

Pierre Morelli, «Marc Augé, Pour une anthropologie de la mobilité. », Questions de communication [En ligne], 17 | 2010, mis en ligne le 23 janvier 2012, consulté le 22 septembre 2020. URL : http:// journals.openedition.org/questionsdecommunication/256 ; DOI : https://doi.org/10.4000/ questionsdecommunication.256

\section{Ce document a été généré automatiquement le 22 septembre 2020}

Tous droits réservés 


\section{Marc AUGÉ, Pour une anthropologie de la mobilité.}

Paris, Payot \& Rivages, coll. Manuels Payot, 2009, 91 p.

Pierre Morelli

\section{RÉFÉRENCE}

Marc AUGÉ, Pour une anthropologie de la mobilité. Paris, Payot \& Rivages, coll. Manuels

Payot, 2009, 91 p.

1 Pour le théoricien de la «surmodernité " (Augé Marc, Non-lieux, introduction à une anthropologie de la surmodernité, Paris, Éd. Le Seuil, 1992), les mouvements de population, conjugués à l'essor que connaît la communication instantanée, contribuent à développer une forme de mobilité surmoderne. Dans un monde par nature inachevé et où naît et perdure le paradoxe d'un cadre " où l'on peut tout faire sans bouger et où l'on bouge pourtant» (p.8), il est plus que jamais essentiel de prendre puis de garder une position critique par rapport à l'idée et à la terminologie dérivées de la globalisation. Dans son dernier ouvrage qui comprend six chapitres, Marc Augé interroge la mobilité et met la notion de frontière à l'épreuve de l'exclusion. Les exemples sur lesquels il s'appuie sont l'urbanisation et le tourisme; ils lui permettent de formuler des perspectives (inventer la ville du futur, penser de la mobilité). Le lecteur déplorera sans doute le manque de précisions concernant certaines sources citées. Mais, si ce parti pris contribue à alléger l'écriture, il aurait été intéressant de rassembler les références dans une bibliographie finale afin d'offrir à la lecture toute la profondeur scientifique que ce type d'ouvrage mérite.

2 L'urbanisation actuelle induit de nouvelles formes de mobilité et trace des frontières poreuses entre centre et périphérie, et entre périphéries elles-mêmes. L'exclusion n'est plus uniquement engagée dans un rapport nord-sud : « Le problème de l'habitat et de la pauvreté urbaine est maintenant présent au cœur des plus importantes mégalopoles occidentales » (p. 26). Des quartiers privilégiés de villes africaines ou sud-américaines 
sont directement liés aux réseaux mondiaux alors que la précarité et la clandestinité envahissent des zones « déqualifiées » dans les villes occidentales, fruit de l'instabilité, «version noire de la mobilité que l'on associe aux aspects les plus dynamiques de l'économie » (p. 27). Pour l'auteur, l'urbanisation du monde exprime donc «toutes les contradictions du système de globalisation » (p. 32). Plus encore, si le «monde est une ville» parce qu'il représente l'idéal et l'idéologie du système de la globalisation, la "ville est un monde " où "s'expriment les contradictions ou les tensions historiques engendrées par ce système " (p.34). Or, la coexistence monde-ville et ville-monde génère des zones intermédiaires qui, étant vides, établissent des points aveugles pour le regard des habitants de la ville. À travers ces zones vides constituant « la face invisible de la mondialisation [...] que nous ne pouvons, que nous ne voulons et ne savons pas voir » (ibid.), le lecteur averti retrouvera avec intérêt ces "non-lieux » qui avaient été théorisés dans l'ouvrage éponyme précédent. La frontière devient alors sociologique, elle instaure une coupure et engage plusieurs niveaux d'exclusion avec les "clandestins", entre immigrés officiels et "sans papiers», jusqu'aux enfants d'immigrés nés en France, victimes du chômage et d'une scolarité défaillante. L'auteur constate que l'aveuglement des regards s'intensifie alors qu'apparaissent, reviennent et "se contaminent » les mots "exclusion", "clandestin », "sans-papiers ", au risque d'enfanter «de nouvelles peurs et violences potentielles» (p. 38). Ce qui a pour effet d'augmenter l'exclusion et de dresser de nouvelles frontières.

Dans un chapitre central, Marc Augé convoque deux figures radicalement différentes du voyageur qu'il oppose : l'ethnologue, le touriste consommateur. Voyageant seul et pour une longue durée, le premier cherche par le dépaysement à entreprendre un voyage « hors de lui-même » (p. 62) tandis que le second, maintenant l'expérience dans une proximité avec son cadre habituel, "reste chez lui, même lorsqu'il est ailleurs » (p.59). En effet, le touriste moderne compile les expériences qu'il peut sélectionner sans réel projet préalable, à partir de l'affichage dans des catalogues et sans prendre de précaution particulière, de destinations potentielles entassées, banalisées et réduites à l'état de produits à consommer dans l'urgence. Bercé par l'illusion de se croire voyageur, il ne peut percevoir que c'est l'idée de voyage qui est en ruine (p. 58).

Pour Marc Augé, l'étude du tourisme contemporain est non seulement utile pour l'analyse anthropologique de la mobilité, mais elle offre également l'occasion de rappeler au lecteur les lignes forces, les principes du travail et de la méthodologie ethnologique. Parce qu'elle apporte au regard une efficacité particulière dans «le monde d'aujourd'hui, dans lequel [...] les notions de centre et de périphéries sont en crises » (p. 69), l'expérience ethnologique se placerait plus que jamais dans le champ de la modernité. Les nouvelles formes de migrations sont également examinées à travers les tendances actuelles de l'urbanisme. Le déplacement physique de l'exode rural se prolonge par un "déplacement de l'utopie » (p. 76). À trop étendre son périmètre, le flux d'habitants qui ne cesse d'alimenter le développement de la ville semble paradoxalement la faire disparaître (p. 77). D'où l'hypothèse du décentrementformulée par Marc Augé - de la mise en relation avec l'extérieur, au niveau méta (ville, monde) et à celui de l'individu appareillé par les technologies de l'information et de la communication qui maintiennent ce dernier en relation permanente avec l'extérieur.

5 Marc Augé reprend ensuite une position énoncée dans Non-lieux, introduction à une anthropologie de la surmodernité. Surmodernité et postmodernisme constitueraient les revers opposés d'une même pièce, positif à l'excès pour la première et négatif pour le 
second. Il constate que les prévisions pessimistes de Jean-François Lyotard (La Condition postmoderne : rapport sur le savoir, Paris, Éd. de Minuit, 1979) et, plus précisément, son annonce de la fin des grands récits, semble être prise en défaut. La fin de l'histoire n'étant finalement pour l'auteur ni plus ni moins qu'un "nouveau "grand récit" " actuel (p. 86). L'auteur n'oublie pas d'adresser une critique à la pensée contemporaine, incapable d'organiser le "monde humain coextensif à la planète toute entière)" (p. 87). Rappelant que l'histoire française contemporaine a montré que la mobilité ne relève pas uniquement d'une problématique de l'espace, Marc Augé pose l'idée selon laquelle "penser la mobilité, c'est aussi apprendre à repenser le temps" (ibid.); une option que seule l'éducation peut permettre. En conclusion de l'ouvrage, la nouvelle forme d'utopie que l'auteur appelle de ses vœux consiste à s'affranchir de soi et de son entourage, et à se libérer du joug de toute culture particulière au bénéfice d'une approche transculturelle de l'individu (p. 91).

\section{AUTEURS}

\section{PIERRE MORELLI}

CREM, université Paul Verlaine-Metz

morelli@univ-metz.fr 